\title{
Financial Account
}

National Cancer Institute

\section{Source}

National Cancer Institute. Financial Account. NCI Thesaurus. Code C88186.

A formal relationship established to provide for regular banking and business services. 